\title{
Lefispedia: el alcance mediante Internet de una experiencia docente de trabajo colaborativo
}

Lefispedia: the impact throughout Internet of a collaborative-work teaching experience

\author{
Fernando Galindo Ayuda (1), Pilar Lasala Calleja (2)
}

(1) Departamento de Derecho Penal, Filosofía del Derecho e Historia del Derecho, Facultad de Derecho, Universidad de Zaragoza, cfa@unizar.es (2) Departamento de Métodos Estadísticos, Facultad de Ciencias, Universidad de Zaragoza, lasala@unizar.es

\begin{abstract}
Resumen
Se presenta la experiencia realizada entre 2008 y 2012 en la construcción de una Wikipedia que consta de conceptos y términos de carácter jurídico y tecnológico especialmente. Dado que la Wikipedia, que tiene el nombre de Lefispedia, es accesible por Internet, en este trabajo se muestran características generales de la información que contiene, datos relevantes sobre su método de construcción y la repercusión social alcanzada por un trabajo planeado inicialmente para un ámbito académico cerrado.
\end{abstract}

Palabras clave: Wikipedia. Lefis. Sistemas documentales. Acceso a información. Internet. Innovación docente.

\section{Marco de la actividad}

LEFIS es el acrónimo de Legal Framework for the Information Society, una red de universidades y centros de investigación que, interesados por la temática que da nombre a la red -el estudio y la docencia de los aspectos jurídicos de la sociedad de la información- se constituyó a finales de los años noventa del siglo XX.

Es una marca europea registrada. Está integrada en la actualidad por 128 instituciones (www.lefis.unizar.es). Mensualmente distribuye una Newsletter entre 340 personas.

A partir de 2007 varios miembros de la red iniciaron la impartición de docencia en forma virtual, a distancia, constituyendo un Campus Virtual compartido sobre Derecho y Tecnologías de la Información (www.lawict.unizar.es), utilizando las posibilidades que ofrecen al respecto las tecnologías de la información y la comunicación. En la actualidad cada institución imparte su respectiva docencia utilizando los recursos desarrollados por el proyecto.

Como herramienta común para la impartición de los cursos fue construido un instrumento que permitía tanto establecer acuerdos entre los profesores responsables de la docencia sobre los contenidos de las disciplinas académicas, como la realización de ejercicios prácticos con-

\begin{abstract}
The paper presents the experience gained between 2008 and 2012 in the construction of a Wikipedia consisting of concepts and terms of legal and technological character. As the wikipedia, which has the name Lefispedia, is accessible via the Internet, this paper shows general characteristics of the information that it contains, relevant information on its method of construction and the social impact achieved by a work originally planned for an academically closed enviroment.
\end{abstract}

Keywords: Wikipedia. Lefis. Information retrieval systems. Access to information. Internet. Teaching innovation.

juntos por los estudiantes que permitieran entrenarlos en competencias profesionales relacionadas con la elaboración de definiciones y explicaciones de términos y conceptos limitadas a mil palabras sobre las materias objeto de los cursos, así como la edición, uso y relevancia de información situada en Internet. El instrumento se denominó Lefispedia (lefis.unizar.es/wiki/ doku.php).

En este trabajo nos centramos en presentar las actividades desarrolladas al respecto por la Universidad de Zaragoza con alumnos matriculados en sus cursos y el eco alcanzado por las mismas en Internet.

\section{Descripción de la actividad}

\section{1. ¿Qué es la LEFISPEDIA}

y qué se pretende con ella?

La Lefispedia es el conjunto de términos y conceptos desarrollado en formato Wikipedia (1) por estudiantes, que da cuenta, en textos limitados (más o menos) a mil palabras, del campo de conocimiento asumido por cada uno de ellos como objeto a definir.

La selección de términos fue realizada en un primer momento por profesores responsables de asignaturas relacionadas con Derecho y Tecnologías de la Información. En total fueron 
seleccionados 76. Completada la definición de estos términos, se tomó como referencia para la selección de nuevos los que integran el tesauro europeo Eurovoc (eurovoc.europa .eu/drupal/ $? q=e s)$, propuestos por los estudiantes como objeto de definición y aceptados por el profesor responsable de la correspondiente asignatura.

Los términos han sido definidos en español y algunos de ellos en inglés, alemán o portugués (brasileño).

Los términos son colocados por el alumno que los desarrolla en la página Web que constituye la Lefispedia atendiendo a las reglas de edición del programa Wikipedia (software libre) que los gestiona.

Los términos, publicados con su nombre por los alumnos responsables de los mismos como sus autores (2), son testeados y calificados por los alumnos utilizando criterios de calificación proporcionados por el profesor.

Se publican en formato de Enciclopedia/Wikipedia ordenados alfabéticamente (3). También lo son en forma sistematizada atendiendo a categorías proporcionadas por documentalistas (4).

Publicadas las definiciones en Internet, alcanzadas por los alumnos las competencias profesionales a las que la actividad conduce (5), lo que también se pretende con la Lefispedia es conocer el eco y efecto que tienen dichas definiciones, una vez que son de público acceso al aparecer en la red de comunicaciones en la que Internet se ha ido constituyendo como el incuestionable medio de transferencia de información que es (6).

Para detallar el contenido de la Lefispedia se expresa a continuación el tipo de alumnos que ha cumplimentado los términos y las materias sobre las que versan. Después se entra a considerar, aportando algunos datos significativos, el seguimiento que la página Web que constituye la Lefispedia ha tenido en Internet.

\section{2. ¿Qué alumnos han participado?}

La Tabla I, en apéndice, da cuenta de que la experiencia ha sido desarrollada por alumnos matriculados en las asignaturas reseñadas en la misma. El número total de alumnos matriculados en la Universidad de Zaragoza que han conocido la existencia de la experiencia ha sido 596. No todos ellos han realizado el estudio de los términos: como se expresa más abajo (2.3) los términos en español existentes son 296, con lo cual sólo el 49 por ciento de los matriculados han hecho propuestas: cada estudiante ha de elaborar un único concepto/término.

\begin{tabular}{|c|c|c|}
\hline Años & Titulación y curso & Alumnos \\
\hline \multirow{4}{*}{$\begin{array}{l}2008- \\
2009\end{array}$} & Licenciado en Derecho. Filosofía del Derecho & 34 \\
\hline & $\begin{array}{l}4 \text { Ingenierías. Ética y legislación para } \\
\text { ingenieros }\end{array}$ & 19 \\
\hline & Administración electrónica. Libre elección & 40 \\
\hline & Administración electrónica G9. A distancia & 29 \\
\hline \multirow{5}{*}{$\begin{array}{l}2009- \\
2010\end{array}$} & Licenciado en Derecho. Filosofía del Derecho & 64 \\
\hline & $\begin{array}{l}4 \text { Ingenierías. Ética y legislación para } \\
\text { ingenieros }\end{array}$ & 15 \\
\hline & Administración electrónica. Libre elección & 46 \\
\hline & Electronic government. Libre elección & 21 \\
\hline & Administración electrónica G9. A distancia & 33 \\
\hline \multirow{5}{*}{$\begin{array}{l}2010- \\
2011\end{array}$} & Licenciado en Derecho. Filosofía del Derecho & 64 \\
\hline & $\begin{array}{l}4 \text { Ingenierías. Ética y legislación para } \\
\text { ingenieros }\end{array}$ & 17 \\
\hline & Administración electrónica. Libre elección & 14 \\
\hline & Electronic government. Libre elección & 7 \\
\hline & Administración electrónica G9. A distancia & 40 \\
\hline \multirow{4}{*}{$\begin{array}{l}2011- \\
2012\end{array}$} & Licenciado en Derecho. Filosofía del Derecho & 49 \\
\hline & $\begin{array}{l}4 \text { Ingenierías. Ética y legislación para } \\
\text { ingenieros }\end{array}$ & 14 \\
\hline & Administración electrónica G9. A distancia & 62 \\
\hline & $\begin{array}{l}\text { Metodología del Derecho. Máster Universitario } \\
\text { en Especialización e Investigación en Derecho }\end{array}$ & 28 \\
\hline Total & & 596 \\
\hline
\end{tabular}

Tabla I. Alumnos matriculados en las asignaturas en las que se ha realizado la experiencia

Como puede verse en la tabla, son alumnos de últimos cursos de licenciatura o ingeniería y una asignatura de un Máster de carácter jurídico. Básicamente son alumnos que realizan una titulación jurídica. También los hay de curriculum técnico como lo son los estudiantes de Ingeniería (Industrial, Química, Informática y Telecomunicaciones), o algunos de los estudiantes de la Asignatura Administración Electrónica del Grupo G9 de Universidades, que es impartida a distancia, y que, como asignatura de libre elección, es realizada por alumnos de cualquier titulación.

La Figura 1 muestra que el número mayor de participantes está constituido por alumnos de Administración electrónica, seguidos por estudiantes de Derecho y finalmente Ingeniería. Ello expresa el marcado carácter interdisciplinar de la experiencia y que el eco que pueda alcanzar en Internet los contenidos ha de ser limitado una vez que los contenidos tienen especialización técnica. 


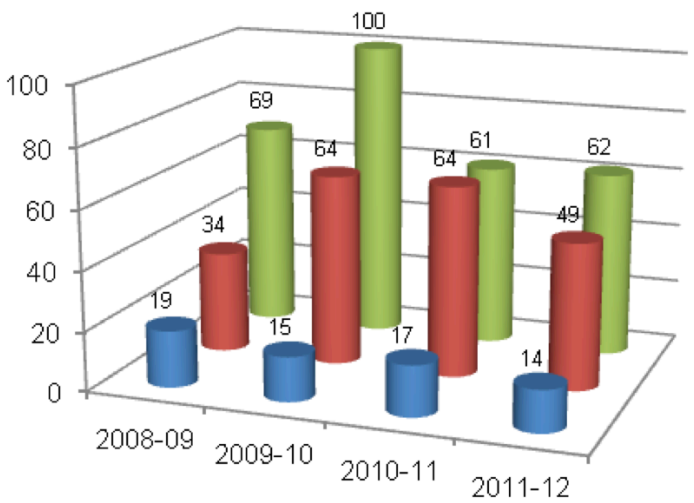

- 4 Ingenierías. Etica y legislación para ingenieros

- Licenciado en Derecho. Filosofía del Derecho

- Administración electrónica (Libre elección. Presencial ya distancia)

Figura 1. Participación de alumnos en algunas asignaturas

\subsection{Estado actual de la LEFISPEDIA}

\subsubsection{Conceptos/términos definidos: en español}

En la actualidad el número total de términos definidos en la LEFISPEDIA es 402, mayoritariamente lo son en español: 296, como puede comprobarse en la Figura 2.

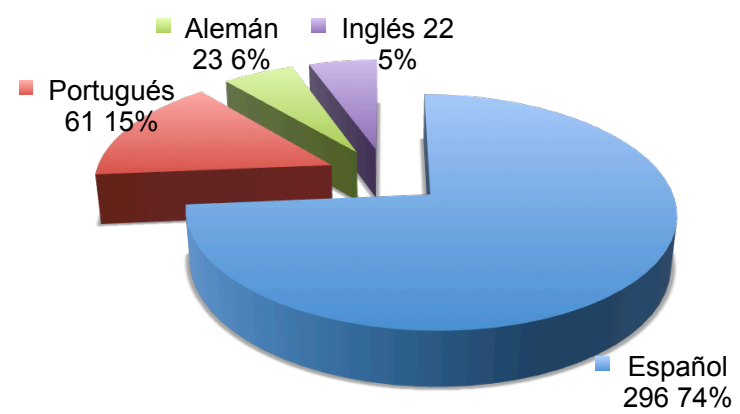

Figura 2. Lefispedia-Desglose del número de términos por idioma

Del trabajo que comporta a los estudiantes la elaboración de los términos da cuenta la Figura 3 que indica el número de veces que fueron editados los términos en los cursos 2010-11 y 2011-12.

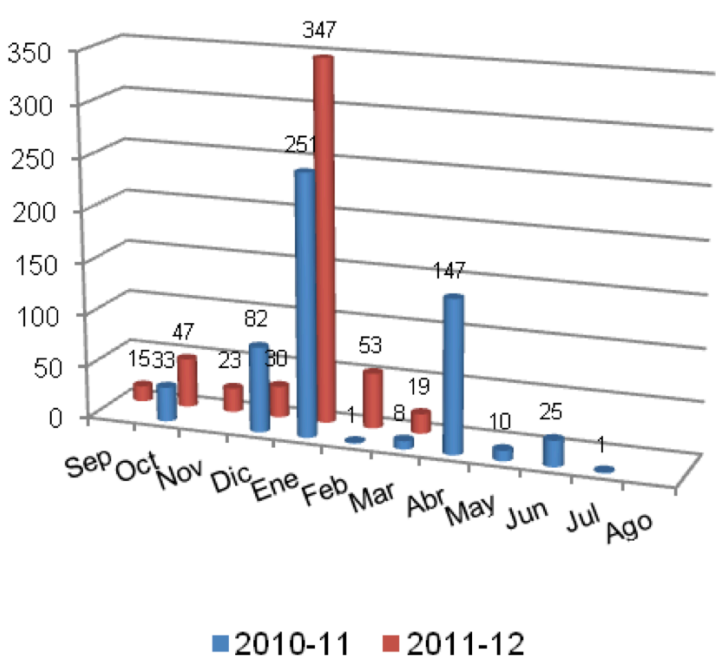

Figura 3. Lefispedia-Evolución de la edición de términos existentes

Cabe observar que fue notable el número de ediciones tanto en uno como otro año: ello es consecuente con la circunstancia de que los estudiantes tienen que acceder en varias ocasiones a la página Web con el fin de diseñar, elaborar y presentar sus trabajos.

Ha de tenerse en cuenta que estos datos son tomados con relación a los resultados que ofrece una versión nueva del programa: recoge estadísticas desde que se instaló la nueva versión del programa (que fue implantada en octubre 2010) y no recoge las de las anteriores.

La ordenación alfabética de los términos en todos los idiomas está presentada en:

http://lefis.unizar.es/wiki/doku.php?do=index\&id=statistics

La lista de conceptos sistematizada por contenidos o materias se hace pública en:

http://lefis.unizar.es/wiki/doku.php?id=es:conceptos

La Figura 4 muestra el número de conceptos por materias. Se destaca que el mayor número de conceptos es el referido a Dominios [aspectos de la vida que son modificados] (138) y a Procedimientos y herramientas [disciplinas y procedimientos que actúan sobre el mundo real](99). El tercer número (30) está constituido por los términos relacionados con perspectiva. 


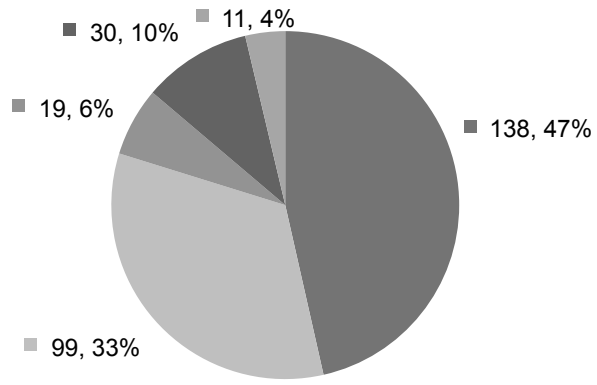

Dominios [Aspectos de la vida que son modificados]

Procedimientos y herramientas [Disciplinas y procedimientos que actuan sobre el mundo real]

Agentes [Actores y entidades]

- Perspectiva

Figura 4. Lefispedia: Número de términos por bloque temático

\section{Dominios}

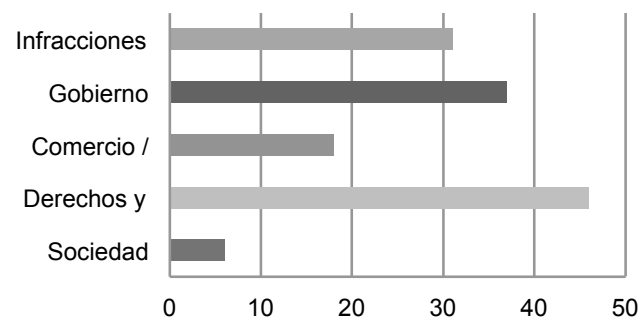

\section{Procedimientos y herramientas}

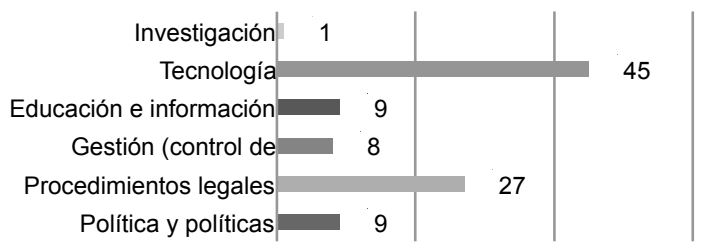

\section{Perspectiva}

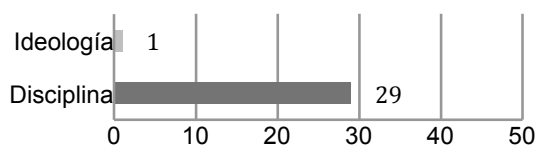

Figura 5. Lefispedia-Desglose del número de términos por bloque

La Figura 5 detalla las subcategorías que integran a cada uno de los tres bloques temáticos que contienen un mayor número de conceptos, dando cuenta del número de conceptos/términos incluidos en cada una de las subcategorías. Puede observarse que, en coherencia con las titulaciones de los estudiantes que elaboran la Lefispedia, el mayor número de conceptos se corresponde con las materias Derechos y Deberes y Tecnología.

\subsubsection{Visitas a la Lefispedia}

Si es importante presentar los conceptos definidos no lo es menos expresar el interés habido por los mismos por las personas y robots que acceden a la Lefispedia por Internet. De ello nos ocupamos a continuación en una primera aproximación. Queda para futuros trabajos la reseña de los términos que son accedidos en mayor o menor medida atendiendo al número de visitantes de las páginas correspondientes a cada concepto/término.

Según la Figura 6, entre el 1 de octubre de 2008 y el 23 de marzo de 2012 el número de visitas a la Lefispedia fue 209.729 , siendo el promedio diario 165. El número de páginas visitadas ha sido 1.451.771, con un promedio diario de 1.143.

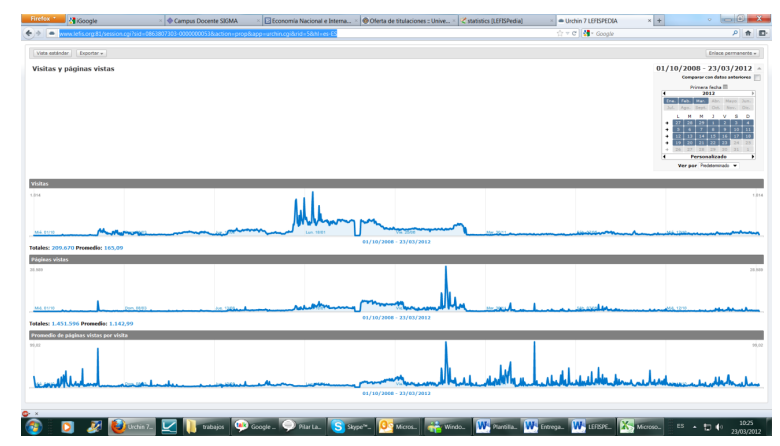

Figura 6. Lefispedia: Número de visitas

Es importante mencionar que la página objeto de consideración: www.lefis.unizar.es/wiki es un subdominio de la página www.lefis.unizar.es y que esta última página tuvo durante el mismo periodo de tiempo considerado 754.318 visitas, con 593 visitas diarias de promedio y 5.130 .464 páginas fueron visitadas siendo el promedio diario de páginas visitadas 4.039 .

En la Figura 6 cabe observar que el mayor número de visitas se produjo a lo largo de 2.009 y 2.010. Es probable que la explicación se deba a que durante esos años la Lefispedia fue accedida, especialmente, a las páginas en inglés y alemán, una vez que a lo largo de esos años se utilizó la herramienta por cursos del Campus Virtual impartidos en el Reino Unido (Belfast), Alemania (Münster), Finlandia (Rovaniemi y 
Vaasa), Torun (Polonia), Portugal (Beja), Turquía (Estambul) y Lituania (Vilnius).

Estudios posteriores determinarán los idiomas de los términos a los que se produjo el acceso. Ello no obstante un indicio de que lo que afirmamos en el párrafo anterior es correcto lo expresan los datos aportados en el siguiente apartado en el que se observa que los visitantes de 2009 y 2010 procedían preferentemente de países en los que el español no es el lenguaje oficial mientras que el acceso desde países donde se habla español sucede en mayor medida en 2011 y 2012.

\subsubsection{Ubicación geográfica de las visitas}

Los datos generales recopilados por el momento indican la ubicación geográfica de quienes han accedido a la información contenida en la Lefispedia (6). Ello queda recogido en las Figuras 7,8 y 9 y en la Tabla II.

Ha de destacarse que las 209.670 visitas a la Lefispedia realizadas durante el periodo aquí considerado (del 1.10.2008 al 23.3.2012) proceden de 177 países. Los diez primeros países de donde proceden las visitas ordenados de mayor a menor ateniendo al número de visitas son: Estados Unidos (63.074), Letonia (Latvia) (53.525), Alemania (13.509), China (11.179), Luxemburgo (8.947), Holanda (7.925), Federación rusa (5.671), España (5.409), Reino Unido (3.303) y Estonia (2.922). Los datos son suficientemente significativos una vez que la suma de todas las visitas hechas a la página desde estos países (175.464) es el 83 por ciento del conjunto de las visitas recibidas.

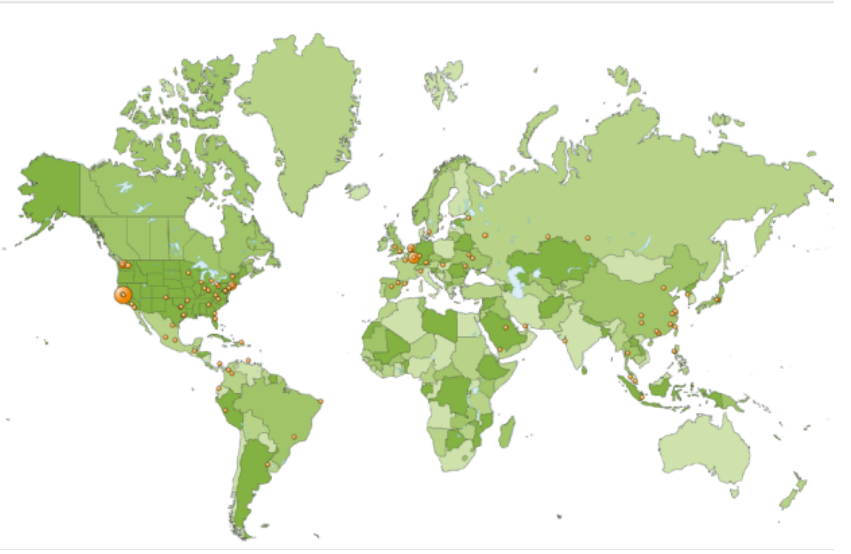

Figura 7. Lefispedia: visitas por ubicación

Por continentes se observa que las visitas procedieron con preferencia de países europeos y de América del Norte (Estados Unidos).

\begin{tabular}{|c|c|c|c|c|}
\hline Año & Total & País & Subtotal 10 & $\%$ \\
\hline \multirow[t]{11}{*}{2009} & 41.835 & Estados Unidos & 15.487 & \\
\hline & & Luxemburgo & 7.309 & \\
\hline & & China & 2.468 & \\
\hline & & Reino Unido & 1.692 & \\
\hline & & Japón & 1.538 & \\
\hline & & Alemania & 1.479 & \\
\hline & & Holanda & 1.261 & \\
\hline & & Canada & 786 & \\
\hline & & Francia & 736 & \\
\hline & & España & 701 & \\
\hline & & Total & 33.457 & $79 \%$ \\
\hline \multirow[t]{11}{*}{2010} & 135.135 & Letonia/Latvia & 53.123 & \\
\hline & & Estados Unidos & 28.361 & \\
\hline & & Alemania & 11.675 & \\
\hline & & China & 7.590 & \\
\hline & & Holanda & 6.637 & \\
\hline & & Federación rusa & 4.513 & \\
\hline & & Estonia & 2.564 & \\
\hline & & Luxemburgo & 1.638 & \\
\hline & & Reino Unido & 1.300 & \\
\hline & & España & 1.255 & \\
\hline & & Total & 118.656 & $87 \%$ \\
\hline \multirow[t]{11}{*}{2011} & 23.582 & Estados Unidos & 13.956 & \\
\hline & & España & 2.253 & \\
\hline & & Méjico & 981 & \\
\hline & & China & 745 & \\
\hline & & Federación rusa & 672 & \\
\hline & & Argentina & 574 & \\
\hline & & Colombia & 538 & \\
\hline & & (no determinado) & 415 & \\
\hline & & Venezuela & 343 & \\
\hline & & Perú & 334 & \\
\hline & & Total & 20.811 & $88 \%$ \\
\hline \multirow[t]{11}{*}{$2012^{*}$} & 8.486 & Estados Unidos & 4.883 & \\
\hline & & España & 1.009 & \\
\hline & & (no determinado) & 713 & \\
\hline & & Ucrania & 410 & \\
\hline & & China & 369 & \\
\hline & & Méjico & 321 & \\
\hline & & Venezuela & 85 & \\
\hline & & Federación rusa & 83 & \\
\hline & & Colombia & 59 & \\
\hline & & Brazil & 57 & \\
\hline & & Total & 7.989 & $94 \%$ \\
\hline
\end{tabular}

Tabla II. Evolución anual de los diez primeros países por número de procedencia de las visitas (*hasta el 23/3/2012) 


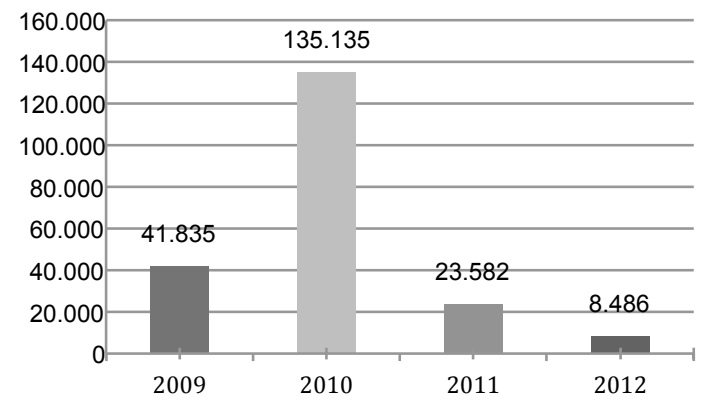

Figura 8. Lefispedia-Total de visitas por año
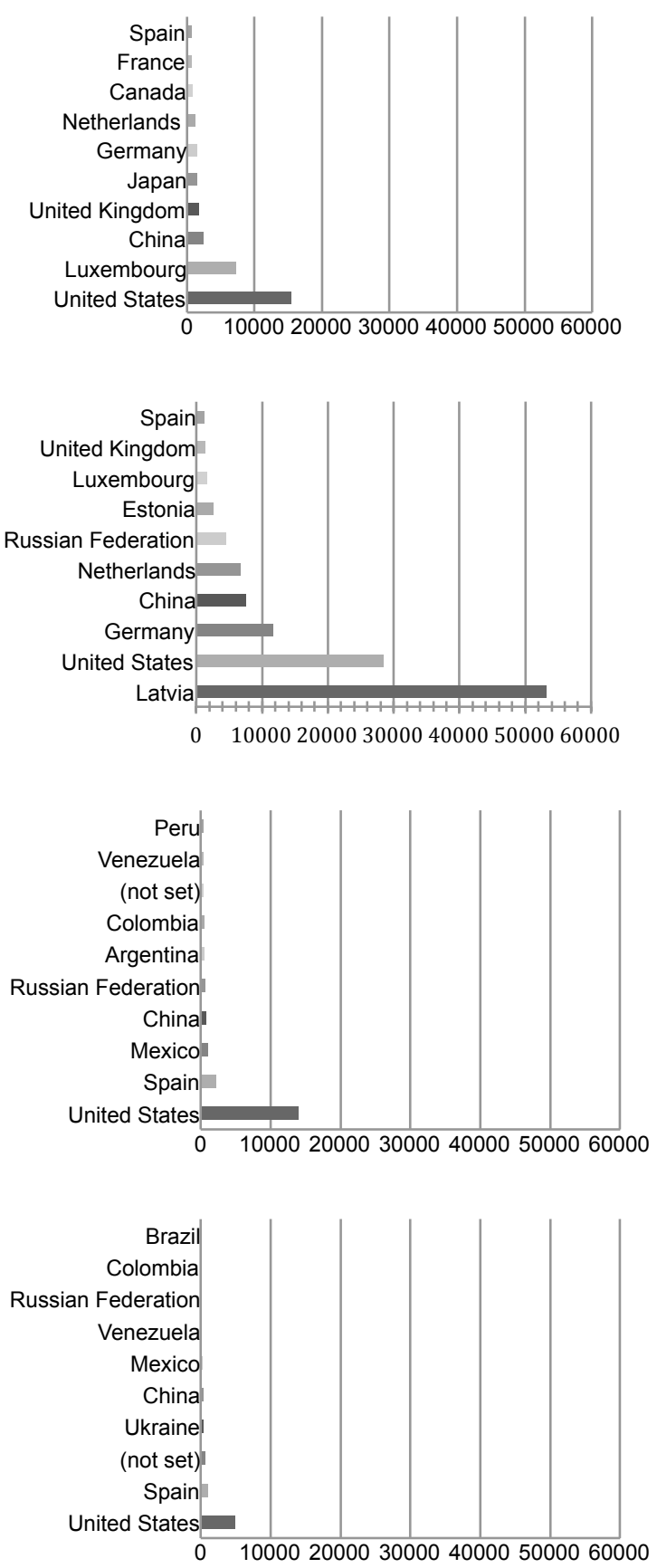

Figura 9. Visitas de los diez primeros países por año
Los números totales alcanzan un significado más concreto si se observan las particularidades de las estadísticas año por año, tal y como lo recogen la Tabla II, en el apéndice, y la Figura 9. La Figura 8 representa gráficamente el número total de visitas por año, observándose la fuerte diferencia que se produce entre los dos primeros años y los dos siguientes. La expresión no determinado indica visitas de las que no consta el país del que proceden.

La Tabla II, en el apéndice, y la Figura 9 muestran que progresivamente se incrementa el número de países origen del mayor número de visitas en los que el español es el idioma hablado. También se reducen las diferencias entre unos y otros países incrementándose la equiparación entre el número de visitas procedentes de diferentes países.

La Tabla II, en el apéndice, concreta el número total de visitas por año expresando los diez primeros países de dónde procedían los visitantes cada año.

Los datos presentados son una muestra de investigaciones a desarrollar en el futuro. Investigaciones que pueden alcanzar más detalladas implicaciones una vez que es posible estudiar mayores particularidades de la compleja información que resumen.

\section{Conclusión}

El trabajo ha puesto de relieve, en una primera aproximación, en qué forma experiencias docentes novedosas en las que se utiliza Internet, pueden ser el comienzo de actividades de investigación, desarrollo e innovación de amplio eco; eco que puede ser medido, salvaguardando siempre la intimidad personal, utilizando las herramientas y recursos que proporcionan las tecnologías de la información y la comunicación, ofreciendo todo ello perspectivas de análisis de actividades sociales que no podían ser tenidas en cuenta hasta la actualidad.

\section{Notas}

(1) El formato de expresión utilizado es adecuado para lo que la experiencia se proponía. Hay que recordar que ha de considerarse a la Wikipedia más un documento producido socialmente que una fuente de información independiente (Royal, 2009).

(2) http://lefis.unizar.es/wiki/doku.php?do=index\&id=statis tics (9 de abril de 2012).

(3) http://lefis.unizar.es/wiki/doku.php?id=es:conceptos (9 de abril de 2012). La clasificación, que parte de las ideas de Ranganathan (1967), se presenta en García, Galindo, Lasala y Ubero (2005). 
(4) No es objeto de este trabajo realizar un balance sobre los logros académicos del proyecto. Estos logros se encuentran recogidos, por ejemplo, en Galindo (2010).

(5) Los autores comparten la idea de que a partir de los trabajos desarrollados con una Wikipedia es posible capturar el cuerpo del conocimiento de un determinado dominio, sin que sea necesaria la elaboración del mismo mediante la acumulación de complejas reflexiones de especialistas (Guo et al., 2009).

(6) Como ya ha sido dicho será objeto de investigaciones realizadas en otros trabajos el estudio de las páginas referidas a los conceptos y términos concretos accedidos.

\section{Agradecimientos}

Este trabajo se desarrolla en el marco de los siguientes proyectos: Establecimiento en Iberoamérica del Observatorio de Gobierno Electrónico. EGOBS, Acción integrada para el fortalecimiento institucional, financiada por la Agencia Española para la Cooperación Internacional al Desarrollo (AECID), 2009-2012; La nueva ecología de la información y la documentación en la sociedad del conocimiento: desarrollo de una métrica sistémica, planificación de un observatorio para su seguimiento e identificación de tendencias básicas y retos estratégicos (infoscopos.com), financiado por la CICYT 2010-2012 (CSO2009-07619); y Mejora en la participación en la sociedad del conocimiento a través de las actividades del Observatorio de Gobierno Electrónico. Aspectos políticos, económicos y empíricos, financiado por la Secretaría General de Universidades del Ministerio de Educación, Cultura y Deportes, convocatoria para la concesión de subvenciones para la cooperación interuniversitaria con Brasil, 2012-2013.

\section{Referencias}

Galindo, F. (2010). Aprendizaje a distancia, redes sociales y go-bierno electrónico: Sobre las posibilidades de la reforma del Espacio Europeo de Enseñanza Superior. // Rover, A., Galindo, F. (eds.). O Governo Eletronico e Suas Mul-tiplas Facetas. Zaragoza: Prensas Universitarias, 2010. 83-120.

García Marco, Francisco Javier; Galindo Ayuda, Fernando; Lasala Calleja, Pilar; Yubero Cortes, Andrés (2005). Towards an Ontology for Knowledge Organisation in the Field of eGovernment. // Kim Viborg Andersen, Åke Grönlund, Roland Traunmüller, Maria Wimmer (Eds.). Electronic Government - Workshop and Poster Proceedings of the Fourth International EGOV Conference 2005, August 22-26, 2005, Copenhagen, Denmark. Linz, Austria: Universitätsverlag Rudolf Trauner, 2005. 158-165.

Guo, T.; Schwartz, D. G.; Burstein Frada; y otros (2009). Codifying collaborative knowledge: using Wikipedia as a basis for automated ontology learning. // Knowledge Management Research \& Practice. 7/3, 206-21

Ranganathan, S. R. (1967). Prolegomena to library classification. Madras: Asia Publishing House.

Royal. C.; Kapila, D. (2009). What's on Wikipedia, and What's Not...? Assessing Completeness of Information. // Social Science Computer Review. 27:1. 138-148.

Santana A., Wood D. J. (2009). Transparency and social responsibility issues for Wikipedia. // Ethics And Information Technology. 11:2. 133-144

Yasseri, T.; Sumi. R.; Kertesz, J. (2012). Circadian patterns of Wikipedia editorial activity: a demographic analysis. // PloS one. 7:1. e30091.

Enviado: 2012-04-27. Aceptado: 2012-08-10. 
